\title{
Donor Status
}

National Cancer Institute

\section{Source}

National Cancer Institute. Donor Status. NCI Thesaurus. Code C159525.

Vital information about a donor at the time of donation. 